\title{
Comparison of isokinetic trunk flexion and extension torques and powers between athletes and nonathletes
}

\author{
Amira Ben Moussa Zouita ${ }^{1, *}$, Fatma Zohra Ben Salah' ${ }^{2}$ Catherine Dziri², Chris Beardsley ${ }^{3}$ \\ ${ }^{1}$ Higher Institute of Sport and Physical Education, Ksar-said, Tunisia \\ 2Department of Medicine Physical and Functional Rehabilitation of the National Institute of Orthopedics "M.T. Kassab", Tunis, Tunisia \\ ${ }^{3}$ Strength and Conditioning Research Limited, London, England
}

This study is aimed to perform and compare maximal concentric isokinetic trunk extension and flexion torques and powers between high-level athletes and a control population. In addition, the ratio of isokinetic trunk extension and flexion torques was measured, and compared between groups. Eighteen high-level male athletes and 15 male nonathletes without low back pain were recruited. Subjects performed isokinetic trunk extension and flexion at $60 \% \mathrm{sec}, 90 \% \mathrm{sec}$, and $120 \% \mathrm{sec}$ through a maximal range of motion in a dynamometer. Trunk extension torque of athletes was significantly higher than in nonathletes at $60^{\circ} / \mathrm{sec}$ and $90 \% \mathrm{sec}$ but not at $120 \% \mathrm{sec}$. Trunk extension power of athletes was significantly higher than the control group at $90 \% \mathrm{sec}$ and $120 \% \mathrm{sec}$ but not at $60^{\circ} / \mathrm{sec}$. There was no difference between the athlete and non- athlete groups in respect of trunk flexion torque or power at any angular velocity. Consequently, the ratio of trunk flexion to extension strength was greater in nonathletes than in athletes. Trunk extension and flexion torques tended to decrease, and trunk extension and flexion powers tended to increase, with increasing angular velocity. High-level athletes seem to display preferentially greater trunk extension strength and power in comparison with trunk flexion strength, compared to nonathletes. This could be caused by the use of strength training exercises such as squats and deadlifts, or it may be associated with greater athletic performance.

Keywords: Strength, Performance, Muscle, Training

\section{INTRODUCTION}

Trunk strength likely plays an important role in many areas relating to health and physical performance. Trunk extension strength may play a role in protecting low back pain, potentially by reducing lumbar kinematic variability during gait (Steele et al., 2016). Moreover, the trunk muscles contribute to the spine stability, and data reported by McGill et al. (2003) suggest that an altered trunk flexion-extension strength ratio may be related to back problems.

Flexion and extension of the trunk from a standing position is governed by a complex system involving sensory (ligaments, Golgi tendon organs, receptors in capsules), neural, active (muscle), and passive (bones, discs, and fascia) structures. Consequently, trunk strength is considered essential for the appropriate distribu- tion of external forces and spine loads in young (Zazulak et al., 2007) and adult athletes (Kibler et al., 2006). And trunk strength may directly or indirectly influence athletic performance in competition because of its relationship with core stability. Therefore, trunk strength is potentially also an important factor for various types of physical activity and sports (Hibbs et al., 2008).

Indeed, sporting activity can produce significant loading on the spine. The repetitive mechanical loading on the spines often in positions involving end range of motion and the increased volume of training required for elite athletic performance can lead to tissue overload and injury (Ong et al., 2003). Up to 20\% of all injuries that occur in sports involve an injury to the lower back. However, the low back is subject to different types of loading in various different movement planes in many sports. Sports can load the spine through repetitive impact (e.g., running), by a twisting mo-
${ }^{*}$ Corresponding author: Amira Ben Moussa Zouita

(iD) https://orcid.org/0000-0002-2266-9472

Higher Institute of sport and physical education, Ksar-said, 2010, Manouba, Tunisia Tel: +216-26625032, Fax: +216-70615533,E-mail: amira_zouita@yahoo.fr

Received: October 6, 2017 / Accepted: January 8, 2018
This is an Open Access article distributed under the terms of the Creative Commons Attribution Non-Commercial License (http://creativecommons.org/licenses/by-nc/4.0/) which permits unrestricted non-commercial use, distribution, and reproduction in any medium, provided the original work is properly cited. 
tion (e.g., golf), at an end of a range of motion (e.g., bending to pick up a ball in rugby), and can involve shear loading (e.g., when being tackled in rugby or American football) or compressive loading (e.g., when weightlifting). Moreover, some types of spine load are widely believed to be more likely to cause injury to the low back than others (McGill et al., 2003).

Isokinetic dynamometry has been one of the most widely used approaches to measure the strength of trunk muscles. Previous studies have reported using dynamometers to measure trunk flexion and extension strength and trunk flexion to extension ratios at various angular velocities and in differing contraction modes (Yahia et al., 2011). Isokinetic measurement has good reliability and precision (Mueller et al., 2011), and is often used for dynamic strength measurement to understand the mechanical profile of skeletal muscle, including trunk muscles. In addition, the biomechanical characteristics of spinal movement have been investigated using isokinetic testing measurement during trunk flexion-extension (Van Damme et al., 2013).

In healthy subjects, trunk strength is typically greatest in sagittal plane extension followed by sagittal plane flexion (Guzik et al., 1996). Several studies have assessed trunk flexion and extension torques in untrained male and female adults (Thompson et al., 1985). The mean of the trunk extension torques from these investigations is $208 \mathrm{Nm}$ (range, 121-360 Nm) and the mean of the trunk flexion torques is $176 \mathrm{Nm}$ (range, 111-296 Nm), respectively with a ratio of trunk flexion to extension of 0.84 (range, $0.54-1.16$ ). From these investigations, it is clear that athlete tend to show the highest trunk strength values, but also the smallest ratio of trunk flexion to extension (Yahia et al., 2011). Elite athletes show a capacity of between 150-240 Nm for trunk flexion and between 200-450 Nm for trunk extension (Baur et al., 2010). Ratios of trunk flexion to extension in healthy untrained adults usually range between $0.7-0.9$ but in athletes, the ratio tends to be between $0.5-0.7$, which occurs in tandem with increased trunk extensor strength (Mueller et al., 2011). However, few previous investigations have directly compared trunk flexion and extension strength and ratios between athletes and nonathletes, and explored the impact of angular velocity on trunk flexion and extension strength and ratios.

The purpose of this study was therefore to perform a case-control investigation in order to compare maximal concentric isokinetic trunk extension and flexion torques and powers between high-level athletes and a control population. In addition, the ratio of isokinetic trunk extension and flexion torques was measured, and compared between groups.

\section{MATERIALS AND METHODS}

\section{Participants}

A case group of 18 high-level male athletes were recruited from various sports (boxing [n=8], wrestling [n=5], weightlifters [ $n=5]$ ), aged $23.3 \pm 2.71$ years, height $1.79 \pm 0.09 \mathrm{~m}$, body mass $74.1 \pm$ $3.81 \mathrm{~kg}$. Additionally, a nonathlete control group was recruited, comprising 15 male athletes aged $22.3 \pm 1.3$ years, height $1.74 \pm$ $0.21 \mathrm{~m}$, mass $74.7 \pm 1.2 \mathrm{~kg}$. The nonathlete group were recreationally active, but did not participate in a sport more than 2 days per week. They performed occasional physical activity, such as running or playing soccer, volleyball, or basketball, but only at a recreational level. Subjects suffering from low back pain during the familiarization process with the dynamometer were excluded. Initially, 23 high-level athletes were recruited and screening for pain during the familiarization process led to a dropout of 5 athletes. The study was approved by the ethics committee and that all subjects provided informed consent. The research reported in this paper was undertaken in compliance with the Helsinki Declaration and the International Principles governing research on humans and animals.

Initially, a medical check-up was performed to confirm that all participants were free from injury and of adequate health to perform the strength testing. Subsequently, anthropometric data were recorded, including body mass and body height. Prior to testing, the subjects undertook a general physical warm-up, comprising $\geq 10$ min on a treadmill, before trunk strength testing. Since the subjects had no previous experience with isokinetic trunk strength testing, the protocol began with a 90-sec warm-up and familiarization trial of isokinetic trunk flexion and extension at a moderate intensity (described below).

For the trunk strength measurement protocol, subjects were tested in a seated position with their anterior superior iliac spines in alignment with the fixed axis of rotation of the Biodex unit. The reliability of the Biodex System 3 has previously been assessed (Secchi et al., 2010). From a practical standpoint, muscle assessment methods using an isokinetic dynamometer are considered to be valid and very reliable, with correlation coefficients of 0.93-0.99 for peak force values and between 0.91-0.96 for total workload values (Guilhem et al., 2014).

The height of the foot plates was adjusted to provide $15^{\circ}$ of knee flexion and the sacral pad was subsequently placed to maintain alignments of the axes of rotation. Four stabilization straps were placed across each subject. One went over both hips and one went across the proximal thighs. The range of flexion and extension that 
each subject moved through was set according to their maximal available range of motion. According to Grabiner and Jeziorowski (1991) ranges of trunk motion no larger than $50^{\circ}$ isolate lumbar motion, and reduce hip flexion and hip extension involvement. The subject was instructed to grasp the shoulder straps during testing. The subjects' feet were secured in straps to a platform that could be adjusted to produce a consistent hip and knee position.

A warm-up and familiarization trial was performed to help the subjects become familiar with the equipment and the testing protocol. The warm-up and familiarization trial consisted of 1 set of 10 consecutive, submaximal concentric trunk flexion and extension repetitions at $120^{\circ} / \mathrm{sec}$ with a 1 -min rest between sets. Each repetition started from the flexion position. After the warm-up and familiarization, subjects performed maximum concentric isokinetic trunk flexion and trunk extension for 5 repetitions at $60 \% \mathrm{sec}$, for 10 repetitions at $90 \% \mathrm{sec}$, and for 15 repetitions at $120 \%$ sec. Verbal encouragement was given throughout the entire test to ensure participants' maximum effort. Peak torque in trunk flexion and trunk extension normalized to body mass, and the ratio of maximum trunk flexion to extension torques, were calculated and expressed as a percentage.

Data were analyzed with IBM SPSS Statistics ver. 20.0 (IBM Co., Armonk, NY, USA) with alpha set at 0.05. Descriptive statistics included the mean, median, minimum, maximum, and standard deviations. For comparisons between groups regarding the quantitative variables was considered the Student $t$-test for independent samples. For intragroup comparisons regarding the quantitative variables were considered the Student $t$-test for paired samples.

\section{RESULTS}

In both the athlete and control groups, trunk flexion torque displayed a trend to decrease with increasing angular velocity (Table 1). However, the only significant difference was between $60 \%$ sec and $120 \%$ sec. In the athlete and control groups, trunk extension torque displayed a trend to decrease with increasing angular velocity (Table 1). However, the only significant difference was between $60^{\circ} \mathrm{sec}$ and $120^{\circ} / \mathrm{sec}$ in the athlete group. When comparing between athlete and control groups, the trunk extension torque of athletes was significantly higher than the control group at $60 \%$ sec and $90 \%$ sec but not at $120 \%$ sec. There was no difference between the athlete and control groups in respect of trunk flexion torque at any angular velocity.

In both the athlete and control groups, trunk extension and trunk flexion powers displayed a trend to increase with increasing angular velocity (Table 2). However, the only significant difference was between $60 \%$ sec and $120 \%$ sec in both groups. When comparing between athlete and control groups, the trunk extension power of athletes was significantly higher than the control group at $90 \% \mathrm{sec}$ and $120 \% \mathrm{sec}$ but not at $60^{\circ} \mathrm{sec}$. There was no difference between the athlete and control groups in respect of trunk flexion power at any angular velocity.

In both the athlete and control groups, trunk flexion to extension ratio displayed a trend to decrease with increasing angular velocity (Table 3). However, the only significant difference was between $60 \%$ sec and $120 \%$ sec. When comparing between athlete and control groups, the ratio of trunk flexion torque to trunk extension torque of athletes was significantly lower in athletes than

Table 1. Peak trunk extension and flexion torques at each angular velocity

\begin{tabular}{|c|c|c|c|c|c|c|}
\hline \multirow{2}{*}{ Group } & \multicolumn{3}{|c|}{ Peak trunk extension torque (Nm) } & \multicolumn{3}{|c|}{ Peak trunk flexion torque (Nm) } \\
\hline & $60^{\circ} / \mathrm{sec}$ & $90^{\circ} / \mathrm{sec}$ & $120^{\circ} / \mathrm{sec}$ & $60^{\circ} / \mathrm{sec}$ & $90^{\circ} / \mathrm{sec}$ & $120^{\circ} / \mathrm{sec}$ \\
\hline Athletes & $440.058 \pm 66.242^{a-c)}$ & $433.667 \pm 55.495^{\mathrm{a}, \mathrm{cl}}$ & $345.267 \pm 124.570^{\mathrm{a}, \mathrm{ab})}$ & $297.342 \pm 50.069^{b)}$ & $261.533 \pm 50.607$ & $211.983 \pm 68.816^{b}$ \\
\hline Control & $373.011 \pm 68.124^{\text {a) }}$ & $365.467 \pm 81.913^{\mathrm{a})}$ & $344.167 \pm 70.539^{a)}$ & $249.233 \pm 50.037^{b)}$ & $261.067 \pm 55.893$ & $190.100 \pm 42.236^{b}$ \\
\hline
\end{tabular}

Values are presented as mean \pm standard deviation.

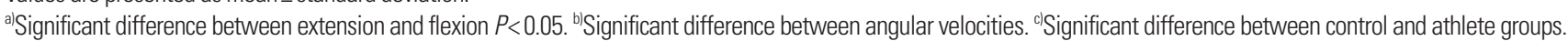

Table 2. Trunk extension and flexion powers at each angular velocity

\begin{tabular}{|c|c|c|c|c|c|c|}
\hline \multirow{2}{*}{ Group } & \multicolumn{3}{|c|}{ Trunk extension power (W) } & \multicolumn{3}{|c|}{ Trunk flexion power (W) } \\
\hline & $60^{\circ} / \mathrm{sec}$ & $90^{\circ} / \mathrm{sec}$ & $120^{\circ} / \mathrm{sec}$ & $60^{\circ} / \mathrm{sec}$ & $90^{\circ} / \mathrm{sec}$ & $120^{\circ} / \mathrm{sec}$ \\
\hline Athletes & $155.183 \pm 42.950^{\mathrm{a}, \mathrm{b})}$ & $269.133 \pm 59.675^{a-c)}$ & $280.500 \pm 60.347^{a, c)}$ & $96.650 \pm 21.409^{b)}$ & $138.958 \pm 24.398^{b /}$ & $146.167 \pm 20.326$ \\
\hline Control & $156.640 \pm 44.874^{\mathrm{a}, \mathrm{bl}}$ & $222.510 \pm 67.379^{a, b)}$ & $231.220 \pm 59.381^{\text {a) }}$ & $109.370 \pm 27.915^{b}$ & $140.020 \pm 30.785^{b /}$ & $158.470 \pm 38.526$ \\
\hline
\end{tabular}

Values are presented as mean \pm standard deviation.

${ }^{\text {a) }}$ Significant difference between extension and flexion $P<0.05$. ${ }^{\text {bl}}$ Significant difference between angular velocities. ${ }^{c}$ Significant difference between control and athlete groups. 
Table 3. Ratio of trunk flexion to extension torque at each angular velocity

\begin{tabular}{lccc}
\hline \multirow{3}{*}{ Group } & \multicolumn{3}{c}{ Ratio $(\%)$} \\
\cline { 2 - 4 } & $60^{\circ} / \mathrm{sec}$ & $90^{\circ} / \mathrm{sec}$ & $120^{\circ} / \mathrm{sec}$ \\
\hline Athletes & $58.6 \pm 10.1^{\text {a) }}$ & $50.1 \pm 10.9^{\text {a) }}$ & $43.9 \pm 9.1^{\text {a) }}$ \\
Control & $66.2 \pm 13.4$ & $69.7 \pm 18.3$ & $52.6 \pm 13.0$ \\
\hline
\end{tabular}

Values are presented as mean \pm standard deviation.

a) Significant difference between nonathlete and athlete groups.

in the control group at all angular velocities.

\section{DISCUSSION}

Our objective was to compare maximal concentric isokinetic trunk extension and flexion torques and powers between high-level athletes and a control population. In addition, the ratio of isokinetic trunk extension and flexion torques was measured, and compared between groups.

In general, there were trends for increasing trunk extension and flexion torques and powers with increasing angular velocity in both groups, although the effect was more marked for trunk extension in the athlete group than in the nonathlete group. Additionally, it was found that the trunk extension torque of athletes was significantly higher than the nonathlete group at $60^{\circ}$ sec and $90 \%$ sec but not at $120 \%$ sec, and also that the trunk extension power of athletes was significantly higher than the control group at $90^{\circ} / \mathrm{sec}$ and $120^{\circ} / \mathrm{sec}$ but not at $60^{\circ} / \mathrm{sec}$. In contrast, there was no difference between the athlete and control groups in respect of trunk flexion power at any angular velocity.

Body posture is an important factor in muscle testing. In our study, isokinetic assessment of trunk was performed in a seated position. The seated position was chosen because the seated position appears to be less affected by the participation of the hip muscles, which decrease the load on the lumbar spine (Morini et al., 2008). This position therefore seems more favourable for a correct evaluation of trunk extension and flexor strength, as well as to express a torque ratio between antagonistic muscle groups. However, while many previous studies have been performed describing the isokinetic evaluation flexor/extensor in a standing position, few have described assessments in a seated position, which leads to difficulty in comparing our findings with the literature (Pinho et al., 2005).

We found that both athletes and nonathletes displayed greater torque and power in trunk extension, at all angular velocities, than in trunk flexion. This is in accordance with previous reports that the trunk extensors are stronger than the trunk flexors (Bernard et al., 2014). We also found that athletes displayed greater trunk extension torque and power than nonathletes, but that there was no difference between athletes and nonathletes in relation to trunk flexion torque and power. This is also in accordance with previous literature, showing that athletic subjects display greater lumbar muscle capacity than sedentary subjects (Iwai et al., 2004).

Muscles typically generate greater concentric force at slow angular velocities, and force decreases as the velocity increases (Rahnama and Bambaeichi, 2008). Our findings similarly indicate that both trunk extension and flexion torques tended to decrease with increasing angular velocity, while trunk extension and flexion powers tended to increase with increasing angular velocity, in both athletes and nonathletes. This likely occurs because of the force-velocity relationship of single muscle fibers, which appears to be in accordance with the torque-angular velocity relationship of single joint motion, at least in some muscles (Hauraix et al., 2017). Even so, few studies have previously assessed the establishing torque-angular velocity and power-angular velocity relationships for the trunk flexor and extensor muscles. Van Damme et al. (2013) found that the angular velocity of isokinetic trunk extension exercises influences the recruitment of the back muscles. Whether this implies that central factors have an influence on the force-velocity profile of trunk extension, however, is unclear. Grimby (1985) suggested that there are different patterns of motor unit recruitment at different speeds in isokinetic measurements, which lead to reduced torque output, because of the different recruitment capabilities of the different muscle fiber types. Indeed, it is also possible that differences in muscle fiber type may have impacted the change in the ratio between trunk flexion and extension with increasing angular velocity, if there is a substantial difference in the type I muscle fiber proportion between the erector spinae and the abdominals, as type II muscle fibers typically faster contraction velocities.

The ratio of the peak torque of the flexors to the extensors can serve as a parameter to assess the muscular balance of a joint. We found that the average trunk flexion to extension ratios varied between $52.6 \%$ to $69.7 \%$ and $43.9 \%$ to $58.6 \%$ respectively, in the nonathlete and athlete groups. Our values seem to be smaller than of literature (64\% to $72 \%$ without gravity correction, according to the authors, or $80 \%$ to $85 \%$ with gravity correction) (Bernard et al., 2014). Simlarly, Simbala et al. (2015) assessed a group of asymptomatic sedentary individuals, and reported a ratio of the peak torque of the flexors to the extensors of $81 \%$ for males. In our study, the ratio of the peak torque of the flexors to the extensors in the athletes group was smaller than in the nonathletes group. This suggests that athletes display preferentially greater 
trunk extension strength and power in comparison with trunk flexion strength, compared to nonathletes. This finding may reflect the use of multijoint strength training exercises among athletes to prepare for sport, as the erector spinae are very highly activated by exercises such as squats and deadlifts. Alternatively, the finding may reflect the important role of the low back in some sporting movements (Aspe and Swinton, 2014) although most mechanical analyses of key sporting activities such as sprinting and jumping have focused on the assessment of joint torques, powers, and work done at the ankle, knee, and hip joints. Even so, specialized muscle development may lead to an imbalance of trunk forces in those sports that clearly involve heavy low back demands, such as weightlifting and wrestling (Secchi et al., 2010). In wrestling, the trunk extensors play a key role in breaking the opponent's balance during forward throw techniques (unbalancing their opponents, moving the opponent's centre of mass in forward and upward direction) for wrestling.

Although not measured in our investigation, the isokinetic trunk flexion to extension ratio may be suitable to evaluate longitudinal changes in trunk muscle function induced by different strength training programs. Specifically designed protocols based on isokinetic trunk testing would thus help coaches in the evaluation of athletes' progression throughout their season or career. Future research should investigate this question.

In a group of athletes and nonathletes, trunk extension and flexion torques and powers tended to increase with increasing angular velocity, although the effect was more marked for trunk extension in the athlete group. Trunk extension torque and power were higher in athletes than in nonathletes, but trunk flexion torque and power did not differ between groups.

Together, these findings suggest that athletes display preferentially greater trunk extension strength and power in comparison with trunk flexion strength, compared to nonathletes. This could be caused by the use of strength training exercises such as squats and dead lifts to prepare for sport, or it may be associated with greater athletic performance. Future research should investigate whether there are associations between trunk extension strength and both squat and deadlift performance, and between trunk extension strength and athletic movements such as sprint running and vertical jumping.

\section{CONFLICT OF INTEREST}

No potential conflict of interest relevant to this article was reported.

\section{REFERENCES}

Aspe RR, Swinton PA. Electromyographic and kinetic comparison of the back squat and overhead squat. J Strength Cond Res 2014;28:28272836.

Baur H, Muller S, Pilz F, Mayer P, Mayer F. Trunk extensor and flexor strength of long-distance race car drivers and physically active controls. J Sports Sci 2010;28:1183-1187.

Bernard JC, Boudokhane S, Pujol A, Chaléat-Valayer E, Le Blay G, Deceuninck J. Isokinetic trunk muscle performance in pre-teens and teens with and without back pain. Ann Phys Rehabil Med 2014;57:38-54.

Grabiner MD, Jeziorowski JJ. Isokinetic trunk extension and flexion strength-endurance relationships. Clin Biomech (Bristol, Avon) 1991; 6:118-122.

Grimby G. Progressive resistance exercise for injury rehabilitation. Special emphasis on isokinetic training. Sports Med 1985;2:309-315.

Guilhem G, Giroux C, Couturier A, Maffiuletti NA. Validity of trunk extensor and flexor torque measurements using isokinetic dynamometry. J Electromyogr Kinesiol 2014;24:986-993.

Guzik DC, Keller TS, Szpalski M, Park JH, Spengler DM. A biomechanical model of the lumbar spine during upright isometric flexion, extension, and lateral bending. Spine (Phila Pa 1976) 1996;21:427-433.

Hauraix H, Dorel S, Rabita G, Guilhem G, Nordez A. Muscle fascicle shortening behaviour of vastus lateralis during a maximal force-velocity test. Eur J Appl Physiol 2017;117:289-299.

Hibbs AE, Thompson KG, French D, Wrigley A, Spears I. Optimizing performance by improving core stability and core strength. Sports Med 2008;38:995-1008.

Iwai K, Nakazato K, Irie K, Fujimoto H, Nakajima H. Trunk muscle strength and disability level of low back pain in collegiate wrestlers. Med Sci Sports Exerc 2004;36:1296-1300.

Kibler WB, Press J, Sciascia A. The role of core stability in athletic function. Sports Med 2006;36:189-198.

McGill S, Grenier S, Bluhm M, Preuss R, Brown S, Russell C. Previous history of LBP with work loss is related to lingering deficits in biomechanical, physiological, personal, psychosocial and motor control characteristics. Ergonomics 2003;46:731-746.

Morini S, Ciccarelli A, Cerulli C, Giombini A, Di Cesare A, Ripani M. Functional anatomy of trunk flexion-extension in isokinetic exercise: muscle activity in standing and seated positions. J Sports Med Phys Fitness 2008;48:17-23.

Mueller S, Mayer P, Baur H, Mayer F. Higher velocities in isokinetic dynamometry: a pilot study of new test mode with active compensation of inertia. Isokinet Exerc Sci 2011;19:63-70.

Ong A, Anderson J, Roche J. A pilot study of the prevalence of lumbar 
disc degeneration in elite athletes with lower back pain at the Sydney 2000 Olympic Games. Br J Sports Med 2003;37:263-266.

Pinho L, Dias RC, Souza TR, Freire MT, Tavares CF, Dias JM. Avaliação isocinética da função muscular do quadril e do tornozelo emidosos que sofremquedas. Rev Bras Fisioter 2005;9:93-99.

Rahnama N, Bambaeichi E. Musculoskeletal assessment in soccer: a review. J Mov Sci Sports 2008;5:13-24.

Secchi LL, Muratt MD, Andrade NV, Greve JM. Isokinetic trunk dynamometry in diferent swimming strokes. Acta Ortop Bras 2010;18:295297.

Simbala M, Czaikoski CK, Eduardo FM, Moser AD, Bernardelli RS. The trunk extensor flexor relationship in men and women by isokinetic dynamometry. Man Ther Posturol Rehab J 2015;13:1-21.

Steele J, Bruce-Low S, Smith D, Jessop D, Osborne N. A randomized controlled trial of the effects of isolated lumbar extension exercise on lumbar kinematic pattern variability during gait in chronic low back pain.
PM R 2016;8:105-114.

Thompson NN, Gould JA, Davies GJ, Ross DE, Price S. Descriptive measures of lsokinetic trunk testing. J Orthop Sports Phys Ther 1985;7:4349.

Van Damme BB, Stevens VK, Van Tiggelen DE, Duvigneaud NN, Neyens E, Danneels LA. Velocity of isokinetic trunk exercises influences back muscle recruitment patterns in healthy subjects. J Electromyogr Kinesiol 2013;23:378-386.

Yahia A, Jribi S, Ghroubi S, Elleuch M, Baklouti S, Habib Elleuch M. Evaluation of the posture and muscular strength of the trunk and inferior members of patients with chronic lumbar pain. Joint Bone Spine 2011; 78:291-297.

Zazulak BT, Hewett TE, Reeves NP, Goldberg B, Cholewicki J. The effects of core proprioception on knee injury: a prospective biomechanicalepidemiological study. Am J Sports Med 2007;35:368-373. 